\title{
A Broadband Noise-Canceling CMOS LNA for 3.1-10.6-GHz UWB Receiver
}

\author{
Chih-Fan Liao and Shen-Iuan Liu \\ Graduate Institute of Electronics Engineering \& Department of Electrical Engineering \\ National Taiwan University, Taipei, Taiwan 10617, R. O. C.
}

\begin{abstract}
An ultra-wideband (UWB) noise-canceling low-noise amplifier (LNA) is presented. By using inductive series and shunt peaking techniques, the effective bandwidth of noise canceling is extended. This LNA has been fabricated in a $0.18 \mu \mathrm{m}$ CMOS process. The measured noise figure is $4.5-5.1 \mathrm{~dB}$ over $3.1-10.6-\mathrm{GHz}$, while the power gain is $9.7 \mathrm{~dB}$ with a $-3-\mathrm{dB}$ bandwidth of $1.2-11.9-\mathrm{GHz}$. It consumes $20 \mathrm{~mW}$ from a $1.8 \mathrm{~V}$ supply and occupies only $0.59 \mathrm{~mm}^{2}$
\end{abstract}

\section{Introduction}

The demand for high-speed wireless communication systems is growing during the last few years. With a frequency spectrum allocated from 3.1 to $10.6-\mathrm{GHz}$, ultra-wideband (UWB) is emerging as a very attractive solution for short-distance and high data rate wireless communications. Two possible approaches have been proposed to implement an UWB system. One uses the multi-band OFDM modulation, while the other transmits short pulses with position or polarity modulation. Although the standard has not been completed, a front-end wideband low noise amplifier is indispensable regardless of the receiver architecture. The amplifier must meet several stringent requirements. Those include broadband input matching to minimize return loss, sufficient gain to suppress the noise of a mixer, low noise figure (NF) to enhance receiver sensitivity, low power consumption to increase battery life, and small die area to reduce the cost.

There are several existing solutions for high frequency wideband amplifiers in CMOS technology. Distributed amplifiers can bring the gain-bandwidth-product (GBW) to a value close to device $f_{T}$, but consume large power and area [1]. Amplifiers employing shunt-shunt feedback are well-known for their wideband matching capability, but require high power consumption to obtain reasonable noise figure [2]. A multi-section LC ladder matching network has been proposed to achieve wideband matching, low noise figure, and low power consumption simultaneously [3]. However, the rapid growth of noise figure at high frequencies decreases the receiver sensitivity when operating at upper bands. Besides, the loss of inductors in the matching network contributes substantial noise, and this makes it difficult to realize them in a small area. In this work, the concept of noise canceling is re-exploited [4]. By using inductive series and shunt peaking techniques and the design methodology described in this paper, broadband noise canceling effectively lowers the noise figure over the target band under reasonable power consumption and small die area.

\section{Circuit Description}

The proposed noise-canceling LNA is shown in Fig. 1.
The input stage incorporates a common-gate topology to facilitate ease of matching. Inductor $\mathrm{L}_{0}$ and parasitic capacitance of M1 and M3 form an LC ladder structure. The noise of M1 is canceled by M2 and M3. The bias of M3 is given by an off-chip RF choke, which also provides the dc current path of M1, and is not shown here for simplicity. Noise current due to M1 flows in opposite directions when combined at the drain of M2 and M3, while the signal currents will be added in phase. Inductors $\mathrm{L}_{1}, \mathrm{~L}_{2}$, and $\mathrm{L}_{3}$ are added to extend circuit bandwidth and can be implemented with narrow traces to save area [5]. An output matching stage is cascaded for testing requirements. Transistor M4, with its source connected to a current source and a capacitor $\mathrm{C}_{\mathrm{hp}}$, forms a high-pass $\mathrm{g}_{\mathrm{m}}$ stage. It filters out low frequency components below $3-\mathrm{GHz}$, and makes the total circuit to exhibit a band-pass frequency response.

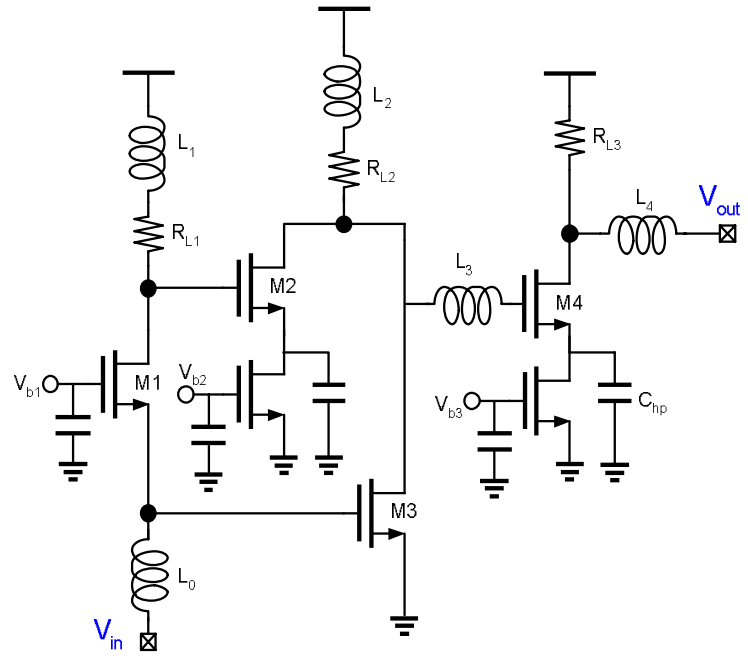

Fig. 1 Proposed broadband noise-canceling LNA.

The overall performance of this circuit lies on the design of the first two stages. The size and bias of M1, M2, and $\mathrm{M} 3$, together with the components value of $\mathrm{R}_{\mathrm{L} 1}, \mathrm{~L}_{0}$, and $L_{1}$, determine the effect of noise canceling and hence the noise figure of this LNA. The following paragraphs describe how to optimize these parameters.

Fig. 2 shows the equivalent circuit for canceling of M1's noise. The capacitor connected to the source of M2 is chosen to be roughly $4 \mathrm{pF}$. It is viewed as a short over the desired band. The input and output are ac-shorted to ground when examining the effect of noise canceling. At low frequencies, the noise current $\mathrm{I}_{\mathrm{n}, \text { out }}$ due to $\mathrm{Ml}$ can be expressed as the following:

$$
I_{n, \text { out }}=\frac{I_{n, M 1}}{1+g_{m 1} R_{S}}\left(g_{m 2} R_{L 1}-g_{m 3} R_{S}\right)
$$


For $\mathrm{I}_{\mathrm{n}, \text { out }} / \mathrm{I}_{\mathrm{n}, \mathrm{Ml}}=0$, which results in successful noise canceling, eq. (2) must be satisfied.

$$
g_{m 2} R_{L 1}=g_{m 3} R_{S}
$$

After noise canceling, the noise figure is then dominant by $\mathrm{R}_{\mathrm{L} 1}, \mathrm{M} 2$, and $\mathrm{M} 3$. The following equations describe how they contribute to the overall noise figure. The input matching condition $\mathrm{R}_{\mathrm{S}}=1 / \mathrm{g}_{\mathrm{m} 1}$ and eq. (2) are assumed through the derivations.

$$
\begin{aligned}
& N F_{R L 1}=\frac{4 k T R_{L 1} g_{m 2}{ }^{2}}{k T R_{S}\left(g_{m 3}+g_{m 2} R_{L 1} / R_{S}\right)^{2}}=\frac{R_{S}}{R_{L 1}} \\
& N F_{M 2}=\frac{4 k T g_{m 2} \gamma / \alpha}{k T R_{S}\left(g_{m 3}+g_{m 2} R_{L 1} / R_{S}\right)^{2}}=\frac{R_{S}}{R_{L 1}} \frac{\gamma}{\alpha} \frac{1}{g_{m 2} R_{L 1}} \\
& N F_{M 3}=\frac{4 k T g_{m 3} \gamma / \alpha}{k T R_{S}\left(g_{m 3}+g_{m 2} R_{L 1} / R_{S}\right)^{2}}=\frac{\gamma}{\alpha} \frac{1}{g_{m 3} R_{S}}
\end{aligned}
$$

Thus, the total noise figure can be approximated as

$$
N F=1+\frac{R_{S}}{R_{L 1}}\left(1+\frac{\gamma}{\alpha} \frac{1}{g_{m 2} R_{L 1}}\right)+\frac{\gamma}{\alpha} \frac{1}{g_{m 3} R_{S}}
$$

From eq. (6), in order to minimize noise contribution from $\mathrm{M} 2$ and $\mathrm{R}_{\mathrm{L} 1}$, the value of $\mathrm{R}_{\mathrm{Ll}}$ should be maximized. To obtain a flat gain response up to $10 \mathrm{GHz}$, smaller size of M1 is then desirable to minimize its parasitic capacitance. Considering the $50 \mathrm{ohm}$ matching condition, the width and bias current of $\mathrm{M} 1$ are chosen to be $30 \mu \mathrm{m}$ and $2.5 \mathrm{~mA}$, respectively. By using inductive shunt peaking, the value of $\mathrm{R}_{\mathrm{Ll}}$ is constrained to voltage headroom, not bandwidth. It heavily depends on the gate bias of M3. Though higher overdrive voltage makes $\mathrm{f}_{\mathrm{T}}$ higher, it drives MOS transistor into velocity saturation region and makes its noise performance worse. Assuming an overdrive voltage around $150-200 \mathrm{mV}$ for $\mathrm{M} 3$, the value of $\mathrm{R}_{\mathrm{L} 1}$ is limited to $180 \mathrm{ohm}$.

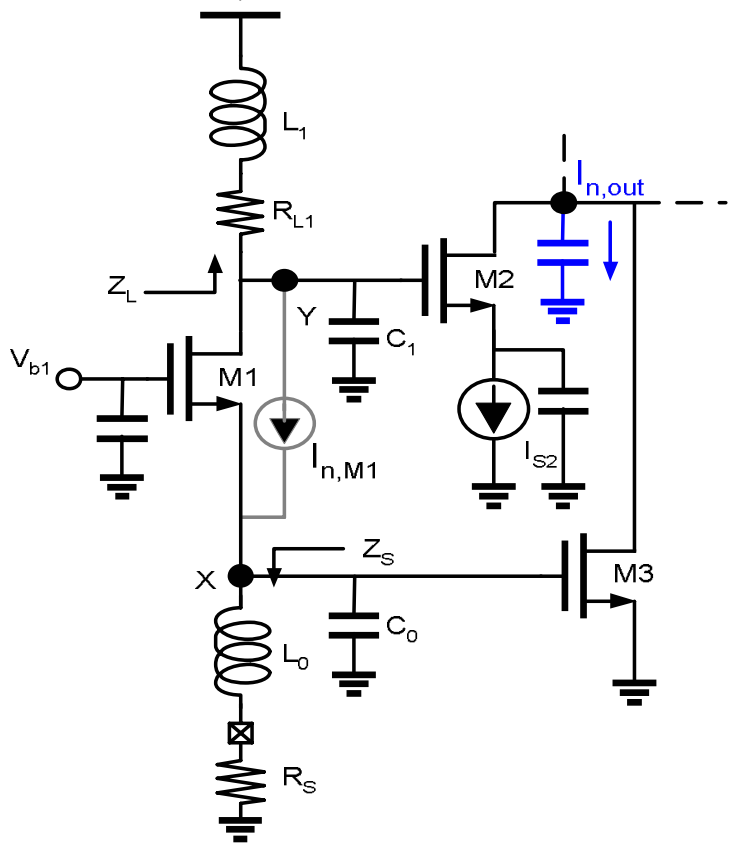

Fig. 2 Equivalent circuit for canceling of M1's noise.
From eq. (2), it is now intuitive to choose M2 as a smaller device and M3 as a larger one. Actually, the size of M2 is chosen considering the trade-off between its parasitic loading on node $\mathrm{Y}$ in Fig. 2 and its noise performance. Similarly, wideband input matching sets an upper limit on the size of M3, while voltage headroom and noise contribution set the lower limit. Inductor $\mathrm{L}_{0}$ and capacitance $\mathrm{C}_{0}$ form an LC ladder structure. When terminated with resistive termination like $1 / \mathrm{g}_{\mathrm{ml}}$, this structure can effectively match to $50 \mathrm{ohm}$ over a wide frequency range. The value of $\mathrm{L}_{0}$ is thus chosen to yield an input impedance of roughly $50 \mathrm{ohm}$ and a high cut-off frequency, while inductor $\mathrm{L}_{1}$ is chosen according to bandwidth optimization. With a total power budget of $20 \mathrm{~mW}$, the only problem now remains to the choice of bias currents of M2 and M3.

The noise canceling condition governed by eq. (2) is not sufficient, since the impedance seen at node $X\left(Z_{S}\right)$ and that seen at node $\mathrm{Y}\left(\mathrm{Z}_{\mathrm{L}}\right)$ depend strongly on frequency. This requires extensive simulations to determine the optimized ratio of $g_{m 3}$ to $g_{m 2}$. Fig. 3 shows the $I_{n, o u t} / I_{n, M 1}$ ratio with different ratios of $\mathrm{g}_{\mathrm{m} 3}$ to $\mathrm{g}_{\mathrm{m} 2}$.

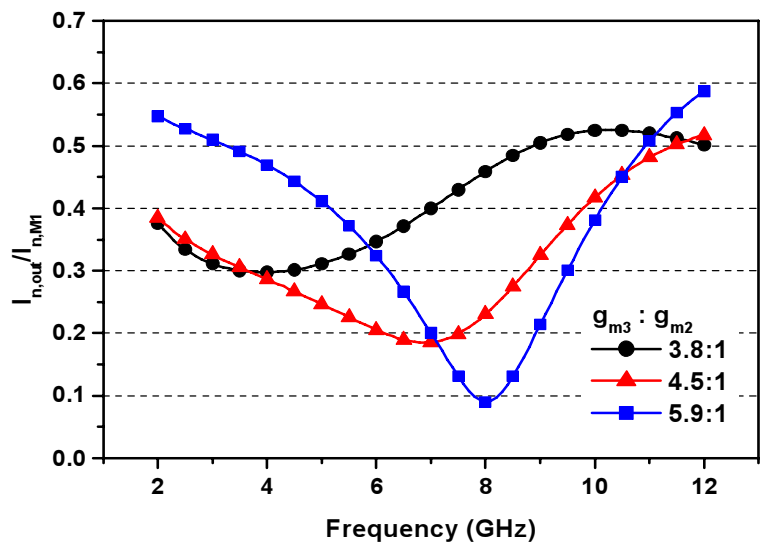

Fig. 3 Simulated $I_{n, o u t} / I_{n, M 1}$ with different values of $g_{m 3} / g_{m 2}$.

It can be seen that the optimized value is not equal to $\mathrm{R}_{\mathrm{L} 1} / \mathrm{R}_{\mathrm{S}}$ due to the frequency dependent nature of $\mathrm{Z}_{\mathrm{L}}$ and $\mathrm{Z}_{\mathrm{S}}$. Parasitic capacitance $\mathrm{C}_{0}$ and $\mathrm{C}_{1}$ cause impedance to roll off at high frequencies, and have been compensated by series inductor $\mathrm{L}_{0}$ and shunt inductor $\mathrm{L}_{1}$, respectively. The series and shunt peaking techniques combined with $\mathrm{g}_{\mathrm{m} 3} / \mathrm{g}_{\mathrm{m} 2}=4.5$ yield broadband noise canceling from 4 to $8-\mathrm{GHz}$. Since the noise power is of primary concern, an $\mathrm{I}_{\mathrm{n}, \text { out }} / \mathrm{I}_{\mathrm{n}, \mathrm{Ml}}$ ratio of 0.3 means that the noise power of $\mathrm{Ml}$ is attenuated by a factor larger than 10 when transferring to output. To further confirm the effect of noise canceling, M3 is turned off and $\mathrm{V}_{\mathrm{bl}}$ is lowered accordingly to emulate the condition without noise canceling. The percentage of M1's noise contributed to total output noise is simulated and compared with the case with noise canceling. As shown in Fig. 4, applying noise canceling substantially lowers noise contribution of the matching device, since it not only cancels noise but also amplifies the signal.

$\mathrm{S}$ parameters and noise figure of this LNA are simulated by Cadence SpectreRF as shown in Fig. 5. 


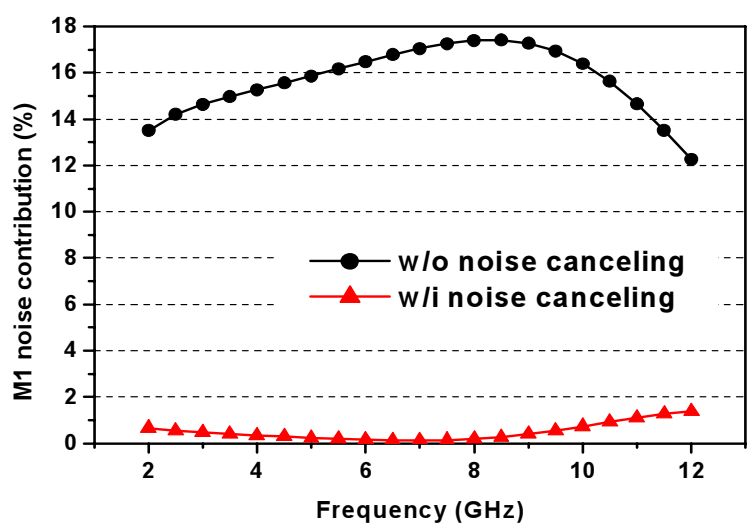

Fig. 4 Simulated noise contribution of M1 with and without noise canceling.

In Fig. 1, due to the large drain to substrate capacitance introduced by M2 and M3 and loading of the last stage, inductors $\mathrm{L}_{2}$ and $\mathrm{L}_{3}$ are used to ameliorate the parasitic capacitance [5]. The output stage is intended for $50 \mathrm{ohm}$ matching. When used in an integrated receiver, resistor $R_{L 3}$ and inductor $\mathrm{L}_{4}$ can be replaced with switching quad to form a single-balanced mixer. Typical loading of I/Q mixers on an LNA is thus considered in this design. The last stage consumes $5 \mathrm{~mA}$ and has $4 \mathrm{~dB}$ loss due to the $25 \mathrm{ohm}$ paralleled resistance. The simulated power gain is $10 \mathrm{~dB}$, and average noise figure over the target band is 3.9dB. Both S11 and S22 are below $-10 \mathrm{~dB}$ up to $11-\mathrm{GHz}$ as shown in Fig. 5.

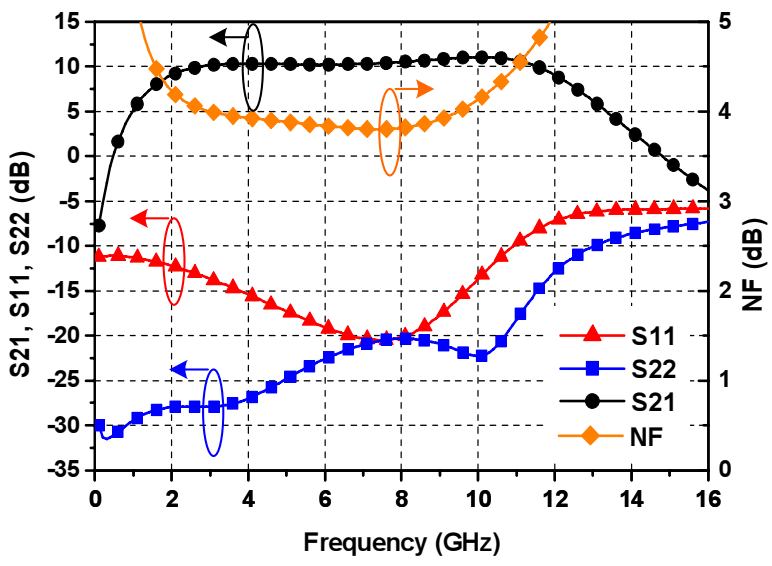

Fig. 5 Simulated S parameters and noise figure.

\section{Experiment Results}

A microphotograph of this LNA is shown in Fig. 6. The circuit has been fabricated in a $0.18 \mu \mathrm{m}$ CMOS process and occupies an area of $0.9 \mathrm{~mm} \times 0.65 \mathrm{~mm}$ including pads. Measurements have been carried out on wafer.

$\mathrm{S}$ parameters and NF are measured using an ATN NP5B measurement system. Fig. 7 shows the measured power gain and output return loss. Measured power gain achieves a maximum of $9.7 \mathrm{~dB}$ at $4.2-\mathrm{GHz}$, and remains 1-dB flatness from 2.4 to $9.4-\mathrm{GHz}$. It also exhibits a $3-\mathrm{dB}$ bandwidth of 1.2-11.9-GHz. The discrepancy between measurement and simulation at high frequencies mainly attributes to insufficient accuracy of inductor and transistor modeling. The measured output return loss is larger than $10 \mathrm{~dB}$ up to $14-\mathrm{GHz}$. It shows different trend at low frequencies compared with simulation. This is due to process variation and the limited frequency range $(1-18-\mathrm{GHz})$ of tuners used for noise measurement in this test plan. The measured input return loss and reverse isolation are shown in Fig. 8. S11 is below $-10 \mathrm{~dB}$ up to $14-\mathrm{GHz}$, while $\mathrm{S} 12$ is below $-35 \mathrm{~dB}$ across the band.

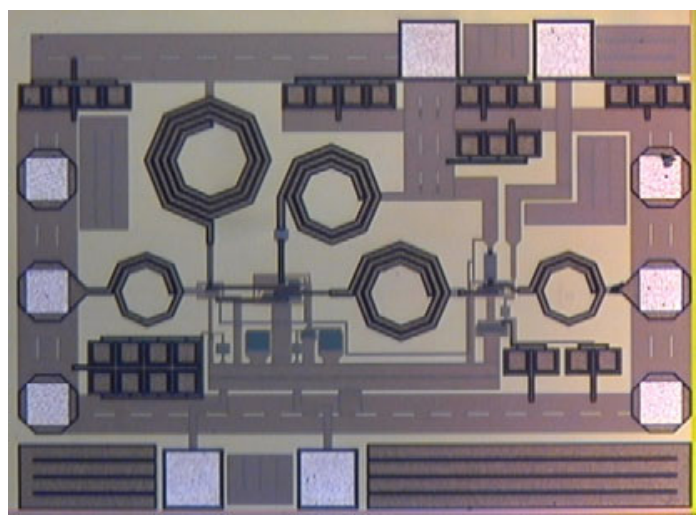

Fig. 6 Microphotograph of the LNA.

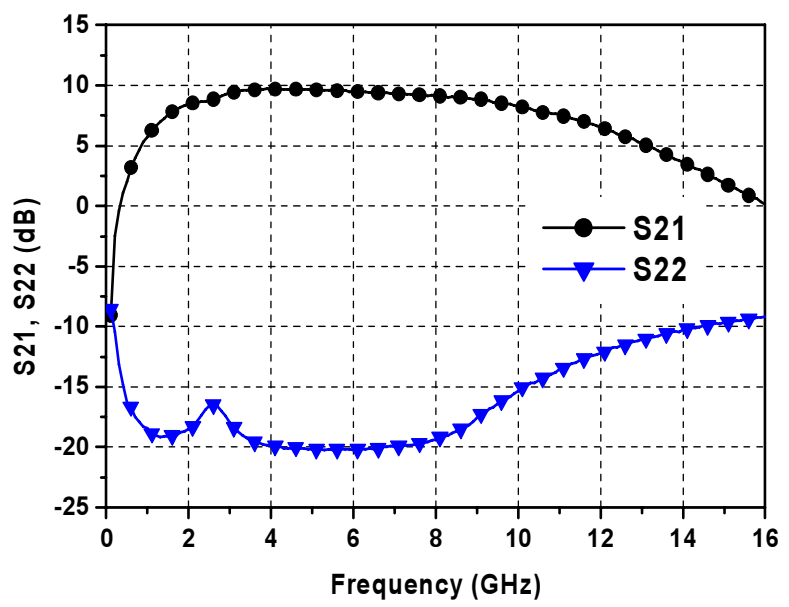

Fig. 7 Measured power gain and output return loss.

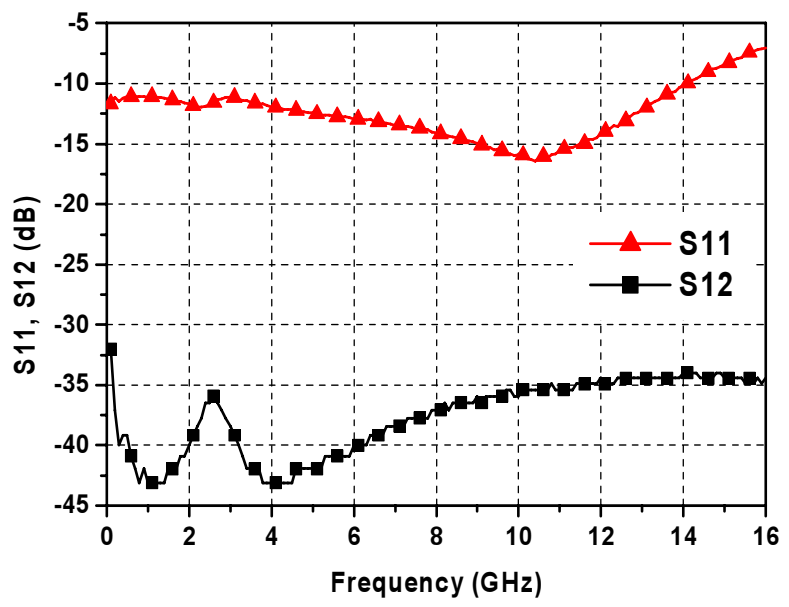

Fig. 8 Measured reverse isolation and input return loss. 


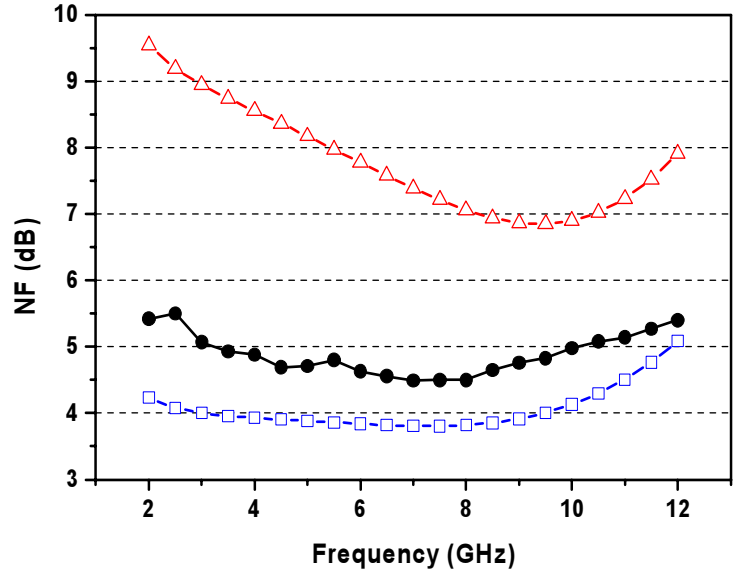

Fig. 9 Measured and simulated noise figures (NFs). $-\Delta-:$ simulated $N F$ without noise canceling $-\square-:$ simulated NF with noise canceling -๑-: measured NF

The measured noise figure is illustrated in Fig. 9 and simulated ones are included for comparisons. Measurement shows a noise figure of $4.5-5.1 \mathrm{~dB}$ over $3.1-10.6-\mathrm{GHz}$, and the average value is $4.7 \mathrm{~dB}$. A slight discrepancy of $0.5-1 \mathrm{~dB}$ is observed between simulation and measurement. This is mainly caused by the lack of noise model of deep submicron CMOS. However, the effect of broadband noise canceling can be confirmed both in simulation and measurement.

Fig. 10 shows the measured IIP3 at 6-GHz. Applying two tones with $1-\mathrm{MHz}$ spacing, the measured IIP3 is $-6.2 \mathrm{dBm}$, while $\mathrm{P} 1 \mathrm{~dB}$ is found to be $-16 \mathrm{dBm}$.

Performance summary of this LNA is listed in Table I. Other previously published LNAs, especially those for UWB systems, are included for comparisons. This work benefits from high bandwidth, low average NF, and small die area with moderate power consumption.

\section{Conclusion}

A broadband noise-canceling LNA for a $3.1-10.6-\mathrm{GHz}$ UWB receiver is implemented in $0.18 \mu \mathrm{m}$ CMOS technology. The proposed circuit achieves a power gain of $9.7 \mathrm{~dB}$, a $3-\mathrm{dB}$ bandwidth of $1.2-11.9-\mathrm{GHz}$, an average noise figure of $4.7 \mathrm{~dB}$ over the target band, and an input matching better than $-10 \mathrm{~dB}$ up to $14-\mathrm{GHz}$. It also occupies a small area of $0.59 \mathrm{~mm}^{2}$ and consumes $20 \mathrm{~mW}$ from a $1.8 \mathrm{~V}$ supply.

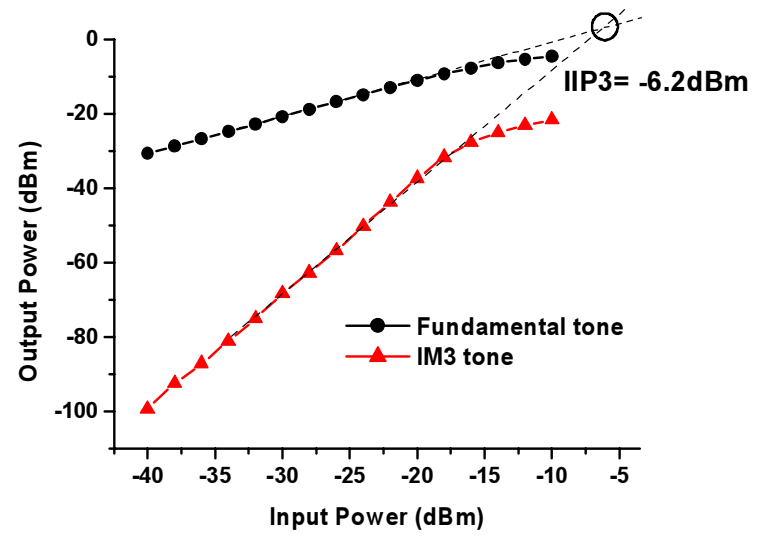

Fig. 10 Measured IIP3 at 6-GHz.

Acknowledgement

This work was supported in part by MediaTek Inc. and National Science Council, Taiwan. The authors would like to thank National Nano Device Laboratory for measurement support and CIC for chip fabrication.

\section{References}

[1] R. Liu, C. Lin, K. Deng, and H. Wang, "A 0.5-14-GHz 10.6-dB CMOS cascode distributed amplifier," Symp. VLSI Circuits Dig. 17, pp. 139 - 140, June 2003.

[2] S. Andersson, C. Svensson, and O. Drugge, "Wideband LNA for a multistandard wireless receiver in $0.18 \mu \mathrm{m}$ CMOS," Proceedings of the 29th European Solid-State Circuits Conference, pp. 655 - 658, Sept. 2003.

[3] A. Bevilacqua and A. M. Niknejad, "An ultra-wideband CMOS LNA for 3.1 to $10.6 \mathrm{GHz}$ wireless receivers," IEEE International Solid-State Circuits Conference, vol. XVII, pp. 382 - 383, Feb. 2004.

[4] F. Bruccoleri, E. A. M. Klumperink, and B. Nauta, "Noise canceling in wideband CMOS LNAs," IEEE International Solid-State Circuits Conference, vol. XLV, pp. 406 - 407, Feb. 2002.

[5] T. H. Lee, The Design of CMOS Radio-Frequency Integrated Circuits, $1^{\text {st }}$ ed., New York: Cambridge Univ. Press, 1998.

[6] C.-W. Kim, M.-S. Kang, P. T. Anh, H.-T. Kim, and S.-G Lee, "An Ultra-Wideband CMOS Low Noise Amplifier for 3-5-GHz UWB System," IEEE Journal of Solid-State Circuits, vol. 40, pp. 544 - 547, Feb. 2005.

[7] R. Gharpurey, "A broadband low-noise front-end amplifier for ultra wideband in $0.13 \mu \mathrm{m}$ CMOS," 2004

IEEE Custom Integrated Circuits Conference, May 2004

Table I. Performance Summary

\begin{tabular}{|c|c|c|c|c|c|c|c|}
\hline & Technology & $\mathrm{BW}_{3-\mathrm{dB}}(\mathrm{GHz})$ & Gain $(\mathrm{dB})$ & $\mathrm{NF}(\mathrm{dB})$ & $\mathrm{IIP3}(\mathrm{dBm})$ & Power $(\mathrm{mW})$ & Area $\left(\mathrm{mm}^{2}\right)$ \\
\hline This work & $\mathbf{0 . 1 8} \boldsymbol{\mu m}$ CMOS & $\mathbf{1 . 2 - 1 1 . 9}$ & $\mathbf{9 . 7}$ & $\mathbf{4 . 5 - 5 . 1 ^ { + }}$ & $\mathbf{- 6 . 2}$ & $\mathbf{2 0}$ & $\mathbf{0 . 5 9}$ \\
\hline$[1]$ & $0.18 \mu \mathrm{m}$ CMOS & $0.5-14$ & 10.6 & $3.4-5.4$ & +10 & $52^{+++}$ & 1.6 \\
\hline$[3]$ & $0.18 \mu \mathrm{m}$ CMOS & $2.3-9.2$ & 9.3 & $4-8^{++}$ & -6.7 & 9 & 1.1 \\
\hline$[6]$ & $0.18 \mu \mathrm{m}$ CMOS & $2.0-4.6$ & 9.8 & $2.3-5.2$ & -7 & 12.6 & 0.9 \\
\hline$[7]$ & $0.13 \mu \mathrm{m}$ CMOS & $\mathrm{x}-5.9$ & 16 & $4.7-5.7$ & $\mathrm{x}$ & $38^{+++}$ & 0.24 \\
\hline
\end{tabular}

${ }^{+}:$Average $=4.7 \mathrm{~dB}$ over $3.1-10.6-\mathrm{GHz} \quad{ }^{++}:$Average $=5.2 \mathrm{~dB} \quad{ }^{++}$: Total power including buffer $\quad \mathrm{x}$ : Not mentioned 